\title{
NOTE
}

\section{THOMPSON: ALL ABOUT FIVE MEN}

\author{
The Editors
}

As of March 25 of this year, the chess world is in possession of the second Volume of Ken Thompson's CDROMs. Together with the first volume it covers nearly all of the endgames of up to five men. Endgames with a Pawn are duly split fourfold. Those able to obtain the CD-ROM need no longer worry about access: ANSI C programs provide an easy entry into this treasury.

For all its mechanical nature, the knowledge so recorded is not trivial. With dismal success theorists have long spread information about endgames, Chéron being the honorable exception. However, all is now revealed beyond a quibble, the information is as clean as whistle and unassailable.

It is fascinating to see how apparently trivial variations lead to radically different solutions.

We can do no better justice to this giant undertaking than by listing sample results. For the endgames in Volume 2 , we identify them, state their maximin, and provide a sample position requiring that number of moves; also stating the player to move (Table 1).

\begin{tabular}{|l|r|l|c|}
\hline Endgame & Maximin & Maximin position & W/B to move \\
\hline KNP(a)KB & 30 & Ke6 Nh2 Pa4; Kh1 Bg2 & W \\
KNP(b)KB & 25 & Kd6 Nf1 Pb5; Kc2 Bf6 & W \\
KNP(c)KB & 23 & Kd1 Nh8 Pc6; Kg1 Ba8 & W \\
KNP(d)KB & 21 & Kb3 Nc3 Pd6; Kh1 Bb2 & B \\
KQNKB & 10 & Ka8 Qb7 Ng8; Kf5 Bd3 & B \\
KRNKB & 26 & Kd8 Rc1 Nb1; Ka3 Bh6 & B \\
KBNKB & 13 & Kc8 Bc4 Ng4; Ka8 Be8 & W \\
KNNKB & 4 & Kd8 Nb5 Nc8; Ka8 Bb7 & W \\
KBP(a)KB & 26 & Kb8 Bd1 Pa2; Kg1 Ba8 & B \\
KBP(b)KB & 36 & Kc3 Bc5 Pb4; Kd1 Bf8 & B \\
KBP(c)KB & 40 & Kc5 Bh7 Pc4; Ka1 Ba4 & W \\
KBP(d)KB & 25 & Ke3 Bh7 Pd5; Kh2 Bg2 & W \\
KQBKB & 9 & Ka8 Qa1 Ba7; Ke6 Bh7 & B \\
KRBKB & 26 & Ka8 Rb6 Ba7; Kc8 Ba4 & B \\
KBBKB & 7 & Kc8 Bd2 Be4; Ka8 Be8 & B \\
KBP(a)KN & 43 & Ka1 Bd7 Pa2; Kg1 Nd8 & B \\
KBP(b)KN & 40 & Ka7 Bh7 Pb2; Ke1 Ne8 & W \\
KBP(c)KN & 35 & Kc4 Bh6 Pc3; Kf2 Ng1 & W \\
KBP(d)KN & 43 & Kc4 Bg1 Pd5; Ka1 Nb3 & B \\
KQBKN & 8 & Ka8 Qg8 Bh7; Kc7 Nc8 & B \\
KRBKN & 21 & Ka8 Rb8 Bc8; Kd8 Na7 & B \\
KBBKN & 67 & Ka8 Bh1 Bh6; Ke4 Ng2 & B \\
KBNKN & 77 & Kc8 Bh7 Ne8; Kh8 Nb7 & W \\
KRP(a)KQ & 7 & Kc7 Rh2 Pa7; Kh4 Qd1 & B \\
KRP(b)KQ & 6 & Kb8 Re5 Pb7; Kb2 Qh7 & W \\
KRP(c)KQ & 9 & Ka7 Rc4 Pc6; Kb2 Qh1 & W \\
KRP(d)KQ & 9 & Ka6 Rh5 Pd7; Kc3 Qg1 & W \\
KQRKQ & 61 & Ka8 Qh8 Rb8; Kg6 Qd8 & B \\
KRRKQ & 15 & Kc8 Rg8 Rh2; Ka1 Qd1 & W \\
KRBKQ & 6 & Kc7 Rb4 Ba4; Ka8 Qf1 & W \\
KRNKQ & 9 & Kd6 Re3 Na6; Kb7 Qa8 & W \\
KNNNK & 22 & Ka8 Na7 Nb8 Ng8; Ke8 & B \\
\hline
\end{tabular}

Table 1: Contents of Thompson's Volume 2 summarized. 


\section{A Few Examples}

We also provide a sample of maximin games, again strictly following Ken Thompson, who supplies complete paths even after conversion (equi-optimal moves are in parentheses).

\section{KNP(a)KB}

1. Ng4 Bh3 2. Kf5 Bf1 3. Kf4 Be2 (Bc4, Ba6, Kg1) 4. Kg3 Ba6 (Kg1) 5. Nf6 (Ne3) Kg1 6. Nd5 Bb7 (Bc4) 7. Nc3 Kf1 8. Kf4 Kf2 9. a5 Ke1 10. Ke3 Ba6 11. Na2 (Nd5) Bb5 (Kd1) 12. Nb4 Kd1 13. Kd4 Kc1 14. Kc5 Bf1 15. Nd5 Kb2 16. Ne3 (Kb4) Ba6 17. Kb4 Kc1 (Kb1) 18. Nc4 (Nf5) Kc2 19. Nd6 Be2 20. Nb5 (Nc4) $\mathrm{Kd} 3$ (Kd2, Kc1, Bd3, Bf1) 21. Kc5 (a6) Ke3 (Kd2, Bf1) 22. Kc6 (Kb6, a6) Kf4 (Bd3, Bc4, Bf1) 23. a6 $\mathrm{Ke} 5$ (Bd3, Bc4, Bf1) 24. Kc7 Bd3 (Bc4, Bf1) 25. a7 Be4 26. Nd6 Ba8 27. Nc4+ Kf6 (Kd4, Kf4, Ke6, Kf5) 28. Kb8 Bc6 (Bd5, Be4, Bf3, Bg2, Bh1) 29. Na5 (Nd6) Bd5 (Be4, Bf3, Bg2, Bh1) 30. Nb7 Bxb7 31. Kxb7 Kg7 (Ke5, Kg5, Kf7, Ke6, Kg6, Kf5) 32. a8Q Kg6 33. Kb6 (Kc7, Kc6, Qa3, Qa4, Qa5, Qf8, Qd8) Kf7 (Kf5, Kg7, Kf6, Kg5) 34. Kc6 (Kc5, Kc7, Qa5, Qg2, Qf3+, Qd5+, Qc6) Ke6 (Kg6, Ke7, Kg7, Kf6) 35. Qa5 Kf7 (Kf6) 36. Qg5 (Kd6, Kd7) Ke6 (Kf8) 37. Qf4 (Kc7) Ke7 38. Qf5 Ke8 39. Kd6 Kd8 40. Qf8\# (Qd7\#)

\section{$\mathrm{KBP}(\mathbf{c}) \mathrm{KB}$}

1. $\mathrm{Kb} 4 \mathrm{Bc} 6$ (Bd7, Be8, Bb3) 2. Ka3 Bd7 (Be8, Bb5, $\mathrm{Bd} 5, \mathrm{Be} 4)$ 3. c5 (Be4) Bc6 4. Bf5 (Bg6) Bd5 (Be4) 5. Bd3 Bc6 (Bb7, Ba8, Be4, Bf3, Bg2, Bh1) 6. Bc4 (Kb3) $\mathrm{Bb} 7$ (Ba8, Be4, Bf3, Bg2, Bh1) 7. Kb3 (Bg8, Bf7, Be6) Be4 (Bf3) 8. Kc3 (Bg8, Bf7, Be6) Bc6 9. Bb3 (Bg8, Bf7, Be6) Bb5 10. Bd5 Bd7 11. Bb7 Bg4 (Bh3) 12. Bh1 (Bg2, Be4, Ba8) Bd7 13. Bd5 Be8 14. Bb7 Bh5 15. Bc8 Bf3 16. Kd4 (Be6) Kb2 17. Be6 Bc6 18. Ke5 Bb7 (Ka3, Kc3) 19. Kd6 (Bd5) Ka3 (Kc3) 20. Bd5 Ba6 21. c6 Kb4 22. Bg2 (Bf3, Be4) Ka5 23. Kc5 Bc8 (Bb5) 24. Bf1 Be6 (Bf5, Bg4) 25. c7 Bf5 (Bg4) 26. Bc4 Bd7 27. Kd6 Bc8 28. Kc6 Kb4 29. Bf1 (Be2, Bd3, Bb5) Ka5 30. Bd3 (Be2) Kb4 (Ka4) 31. Kb6 Bd7 (Be6, Bg4, Bh3, Ka4) 32. Be4 (Ba6, Kb7, Ka7) Bc8 (Be6, Bg4, Bh3, Ka3, Kc3, Kc4, Kb3) 33. Bb7 (Ka7) Bd7 (Be6, Bf5, Bg4, Bh3) 34. Ka7 Ka5 (Kc5, Kb5) 35. Kb8 Kb6 36. Bc8 Bc6 (Bb5) 37. Bh3 (Bg4, Bf5, Be6) Bb7 38. Bf1 Kc6 39. Bg2+ Kd7 (Kb5, Kb6, Kd6, Kc5) 40. Bxb7 (Kxb7) Ke6 (Ke7, Kd6) 41. c8Q+ Kf6 (Ke5) 42. Qc5 (Qf8+, Qg4) Kf7 (Ke6, Kg6) 43. Qg5 (Qf5+, Qe5) Ke6 44. Qf4 (Bd5+, Ba6, Kc8, Kc7) Ke745. Bd5 Kd8 (Ke8, Kd7) 46. Qf6+ Ke8 (Kd7) 47. Bc6\#
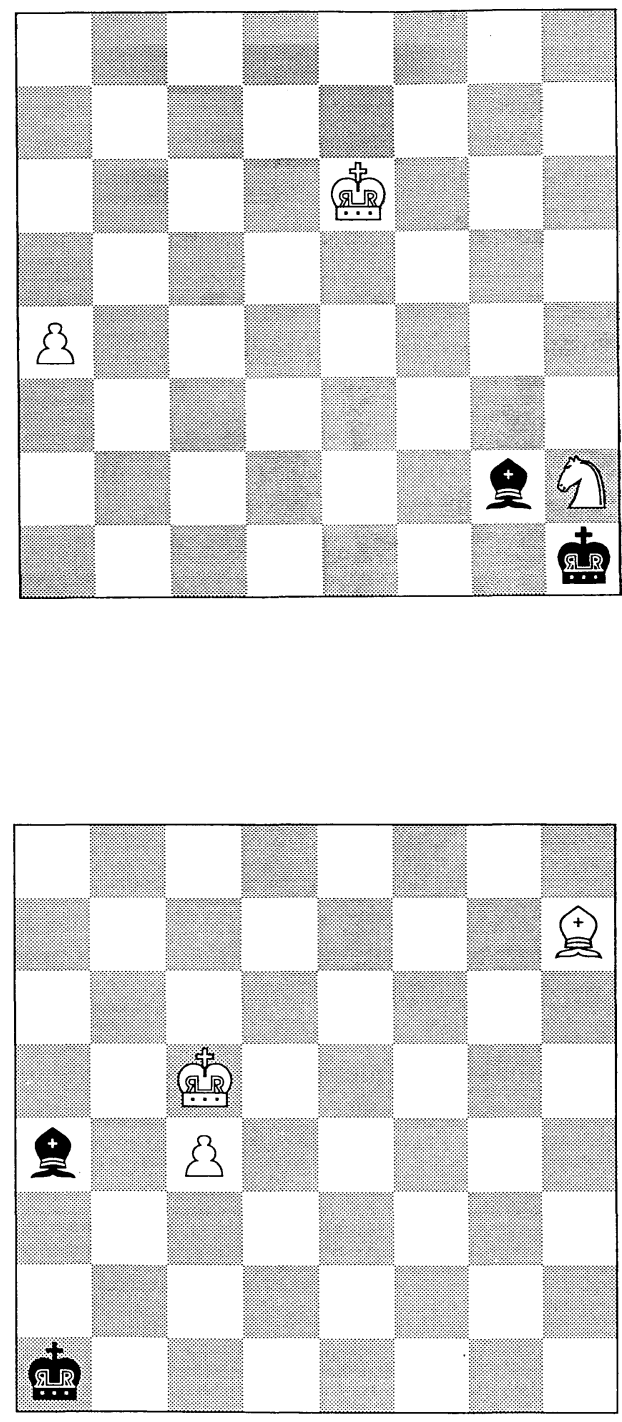

$\mathrm{KBP}(\mathrm{c}) \mathrm{KB}(\mathrm{WTM})$. 


\section{KBP(a)KN}

1.... Kf2 2. Kb2 Ke3 (Kf3) 3. Kc3 Ke4 4. Kc4 Ke5 5. $\mathrm{Kc5} \mathrm{Nb} 7+$ 6. Kc6 (Kb6) $\mathrm{Na} 5+7$. Kb6 Nc4+ 8. Kc5 $\mathrm{Nb} 2$ 9. Bb5 Ke4 10. Kb4 Ke3 11. a3 Kd2 12. Bc6 $\mathrm{Nd} 3+(\mathrm{Ke} 3)$ 13. Kc4 Ke3 14. Kc3 (Bg2) Nc5 15. Bd5 Nd7 16. Bg2 Nc5 17. Bc6 Nd3 18. Ba4 Nc5 19. Bc2 Nd7 20. Bb1 Nb6 21. Ba2 Na4+ 22. Kb3 Nc5+ 23. Kc4 Na4 24. Bb3 Nb2+ 25. Kc3 Nd3 26. Bc2 Nc5 27. Kc4 Nd7 28. Bf5 Nb6+ 29. Kc5 Na4+ 30. Kb4 Nb2 31. Kb3 Nd1 32. Bc2 Nf2 33. Kc4 (a4) Ng4 34. a4 Ne5+ 35. Kb5 Nd7 36. Bf5 Nf6 37. a5 (Be6, Kc5) Nd5 38. Be6 Nc3+ 39. Kb4 (Kc6) Ne4 (Ne2, Nd1) 40. a6 Nf2 41. Ka3 (Ka5, Bf5, Bc4, a7) Kd4 (Kf4, Kd2, Ke4, $\mathrm{Kd} 3, \mathrm{Kf} 3, \mathrm{Ke} 2, \mathrm{Nh} 1, \mathrm{Nd} 1, \mathrm{Nd} 3, \mathrm{Ne} 4)$ 42. a7 Ke5 (Ke3, $\mathrm{Ke} 4, \mathrm{Kd} 3, \mathrm{Nd} 1, \mathrm{Nd} 3, \mathrm{Ne} 4)$ 43. a8Q Kf4 44. Bh3 (Bg4, Bf5, Bd5, Qa5, Qa7, Qh8, Qg8, Qc8, Qg2, Qc6) Ke3 (Nd3, Ne4) 45. Qa7+ Kf3 (Ke2) 46. Bf1 Kg3 (Ng4, Nh1, Nd1, Ne4) 47. Qe3+ Kh4 (Kg4) 48. Qxf2+ Kg5 (Kg4) 49. Bd3 Kg4 50. Qe3 Kh4 51. Qg1 (Qf4+, Bf5) Kh5 (Kh3) 52. Qg3 (Bf5) Kh6 53. Qg6\#

\section{KBP(b)KN}

1. Kb6 Nd6 2. Kc5 Nb7+ 3. Kd4 Nd6 4. Kd5 Nb5 5. Kc5 Nc7 6. Kc6 (Kb6) Na6 7. Kb6 Nb4 8. Kb5 Nd5 9. Be4 Nf6 10. Bc6 Ng4 11. Kc5 Ne5 12. Be4 Nd7+ 13. Kd4 Nb6 (Kd2) 14. b4 Kd2 15. b5 Na4 16. Bf3 Kc1 (Kc2) 17. Kc4 Kc2 18. Be4+ Kb2 19. Kb4 Nb6 20. Bh7 (Bg6) Nd5+ 21. Ka5 Kc3 22. Bg8 Ne7 23. b6 $\mathrm{Nc6}+24 . \mathrm{Kb} 5 \mathrm{Nd} 8$ 25. Kc5 Nb7+ 26. Kc6 Na5+ 27. Kb5 Nb7 28. Bd5 Nd8 29. Kc5 Kd3 30. Bc4+ Kc2 (Kc3, Ke3, Kd2) 31. Ba6 Kb3 (Kc3) 32. Kd6 (Bc8) Ka4 (Kb4) 33. Bc8 Ka5 (Kb4) 34. Kc7 Kb5 35. Bh3 (Bg4, Bf5) Ka5 36. Bf5 (Bg4) Kb5 37. Bd3+ Kc5 38. Bc4 Nc6 39. b7 Nb8 40. Kxb8 Kb6 (Kd6, Kb4, Kd4, $\mathrm{Kc6}, \mathrm{Kxc} 4)$ 41. Kc8 (Ka8) Kc5 42. b8Q Kxc4 43. Qd6 $\mathrm{Kb} 3$ (Kc3) 44. Qd2 (Kc7, Kd7, Kb7) Kc4 (Ka3) 45. Qe3 (Kc7, Kd8, Kb8, Kd7, Kb7) Kb5 (Kd5, Kb4) 46. Qc3 (Qe4, Qd4) Ka4 (Kb6) 47. Qb2 (Kc7, Kb7) Ka5 48. Qb3 (Kc7, Kd7, Kb7) Ka6 49. Qb4 Ka7 50. Qb7\# (Qa5\#)

\section{KBP(c)KN}

1. Kd3 Nf3 2. Be3+ Ke1 3. Bf4 Kd1 4. c4 Ne1+ 5. $\mathrm{Kd} 4 \mathrm{Nc} 2+6 . \mathrm{Kc} 3 \mathrm{Na} 3$ 7. $\mathrm{c} 5 \mathrm{Nb} 5+8$. Kc4 Na7 9. Kd5 Ke2 10. Bb8 Nc8 11. Kc6 Ne7+ 12. Kd6 Nf5+ 13. Kd7 $\mathrm{Nd} 4$ 14. Be5 Nc2 15. Bc3 Na3 16. Bb2 Nc2 17. Kc6 $\mathrm{Nb} 4+18 . \mathrm{Kb} 5 \mathrm{Nd} 5$ 19. $\mathrm{c} 6 \mathrm{Nc} 7+(\mathrm{Ke} 3) 20 . \mathrm{Kc5}$ (Kc4) $\mathrm{Ne} 8$ (Ke3) 21. Be5 Ke3 (Kd2) 22. Kd5 Kd3 23. Bd6 Kc3 24. Be7 Nc7+ 25. Kc5 Ne8 (Kb2, Kd2, Kb3, Kd3, $\mathrm{Kc2}$ ) 26. Bd8 Kb2 (Kd2, Kb3, Kd3, Kc2) 27. Kd5 Ka3 (Kc3, Kb3) 28. Ke6 Kb4 29. Kd7 Kc5 30. Bh4 (Bg5) Kb5 31. Bg5 Kc5 32. Be3+ Kd5 33. Bd4 Nd6 34. c7 Nc8 35. Kxc8 Kc6 (Ke6, Kc4, Ke4, Kd6, Kxd4) 36. Kd8 (Kb8) Kd5 37. c8Q Kxd4 38. Qc2 Ke5 (Ke3, Kd5) 39. Qc4 (Qd3, Ke7) Kf6 (Kf5) 40. Qe4 (Qd4+, Qd5) Kf7 41. Qe5 (Qf5+, Qc6, Kd7) Kg8 (Kg6, Kf8) 42. Ke8 (Ke7) Kh7 43. Qg5 (Kf7) Kh8 44. Kf8 (Kf7) Kh7 45. Qh5\# (Qg7\#)
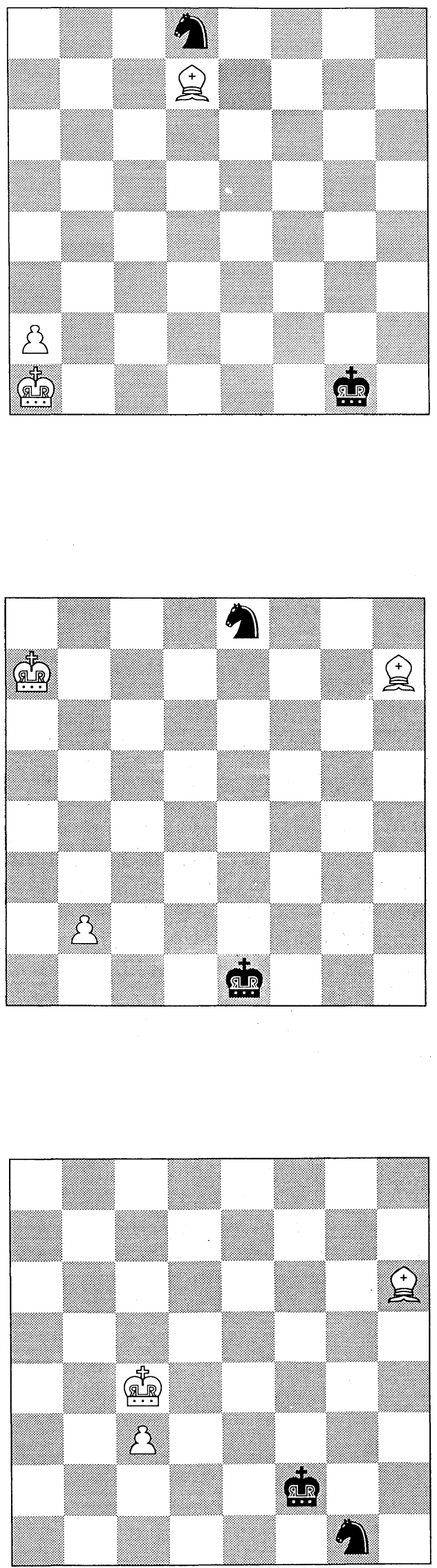


\section{$\operatorname{KBP}(d) K N$}

1. ... Na5+ 2. Kb5 Nb7 3. Kc6 Na5+ 4. Kb6 Nc4+ 5. Kc5 Nd2 6. Kd4 Nf3+ 7. Ke4 Ng5+ 8. Ke5 (Kf5) Nf7+ 9. Ke6 Ng5+ 10. Kf6 (Kd6) Ne4+ 11. Ke5 Nd2 12. Bc5 Kb2 (Ka2) 13. Bb4 Nc4+ 14. Kd4 Nb6 (Kb3) 15. d6 Kb3 16. Kc5 Nd7+ 17. Kb5 Ne5 18. Ba5 Kc2 (Kb2) 19. Kc5 Nd7+ 20. Kc4 (Kc6) Ne5+ 21. Kd5 Nd7 22. Be1 Kb3 23. Kc6 Ne5+ 24. Kc5 Nd7+ 25. Kb5 Nb8 (Kc2) 26. Bf2 Kc3 27. Kc5 Kd3 28. Kd5 Kc3 29. Ba7 Nd7 30. Ke6 Nf8+ 31. Ke7 (Kf7) Ng6+ 32. Kf7 Ne5+ 33. Ke6 Ng6 34. Bg1 (Bf2, Be3) Nf8+ 35. Ke7 Ng6+ 36. Kf7 (Kd7) Ne5+ 37. Ke6 Nc6 38. d7 $\mathrm{Nd} 8+(\mathrm{Kb} 4, \mathrm{Kc} 4)$ 39. Kd6 (Kd5) Kb4 (Kb2, Kc4, Kb3, Kc2) 40. Bb6 Nf7+ 41. Ke6 Ng5+ 42. Ke7 Ne6 (Nf7) 43. Kxe6 Kc3 (Kb5, Kc4, Kb3) 44. d8Q Kc4 (Kc2) 45. Ba5 (Qd1, Qd2, Qd4+, Qd5+) Kb5 (Kb3) 46. Bd2 (Ke5, Kd5, Qd2, Qd5+, Qc7) Ka6 (Kc6, Ka4, Kc5) 47. Kd6 (Kd5, Qc7) Kb7 (Kb5, Ka7) 48. Be3 Ka6 49. Qb6\#

\section{KRRKQ}

1. Rgg2 Kb1 2. Rb2+ Kc1 3. Rbe2 Kb1 4. Rhg2 Qc1+ 5. Kd8 Ka1 (Qd1+) 6. Ra2+ Kb1 7. Rad2 Ka1 8. Rgf2 Kb1 9. Kd7 Ka1 10. Rc2 Qd1+ 11. Kc7 Kb1 (Qb1) 12. $\mathrm{Rb} 2+\mathrm{Ka} 1$ (Kc1) 13. Ra2+ Kb1 14. Rfb2+ Kc1 15. Ra1+ Kxb2 16. Rxd1 Kc2 17. Rd8 (Rd7, Rd6, Rd5) Kb3 (Kc3) 18. Kc6 (Kb6) Kc4 (Kb4) 19. Kb6 (Rd5) Kb3 (Kb4) 20. Kb5 (Kc5, Rc8) Kc3 21. Rd5 (Rd6, Rd7) Kb2 (Kb3) 22. Kb4 (Kc4) Kc2 23. Rd4 (Rd8, Rd7, Rd6) Kb2 24. Rc4 Ka1 (Ka2) 25. Rc2 (Kb3, Ka3) Kb1 26. Kb3 Ka1 27. Rc1\#
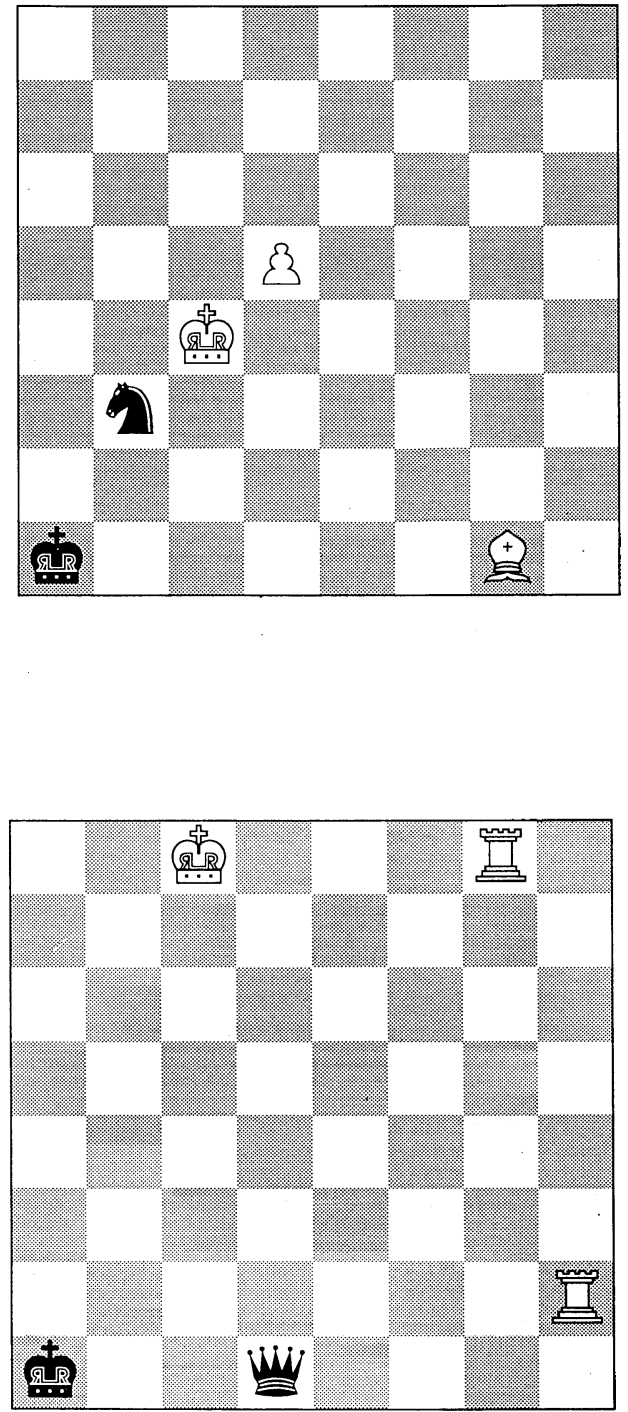

\section{INFORMATION FOR CONTRIBUTORS}

Contributors may be interested to know that the ICCA Journal, as of Vol. 10, No. 1, is a source for the Institute for Scientific Information ${ }^{\circledR}$ (ISI) for inclusion in the CompuMath Citation Index ${ }^{\circledR}$ (CMCI ${ }^{\circledR}$ ), the Automatic Subject Citation Alert (ASCA ${ }^{\circledR}$ ) and SCISEARCH ${ }^{\circledR}$, ISI's on-line database. Author abstracts are now also available in Current Contents on Diskette $₫$, and in on-line files such as SCISEARCH® and Current Contents Search $\otimes$. Moreover, the Journal is also a source for the Information Company R.R. Bowker for inclusion in the International Serials Database which is a source for Ulrich's International Periodicals Directory and the DIALOG on-line service.

Being included in the CMCI ${ }^{\circledR}$, the ICCA Journal is one of the 400 Journals in mathematics, computer science, statistics, operations research, and related disciplines which is abstracted and/or indexed and/or available as tearsheets; this means that the Journal now is accessible in (on-line) database form.

\section{Submission of material}

Contributions to the Journal are welcomed in any form, although preferably by E-mail or on an MS-DOS formatted 5.25 or 3.5 inch diskette. In case contributors prepare their manuscripts with high-quality wordprocessors, it should be noticed that text-files in VENTURA, TEX or TROFF format are processable directly by the Editors, thereby alleviating their task considerably. 